RICYDE. Revista Internacional de Ciencias del Deporte doi: $10.5232 /$ ricyde

Rev. int. cienc. deporte

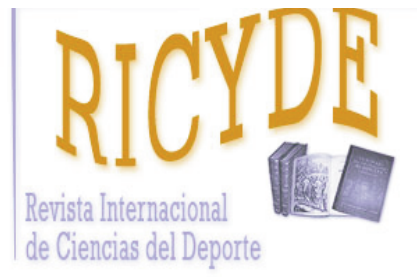

RICYDE. Revista Internacional de Ciencias del Deporte VOLUMEN XIII - AÑO XIII

Páginas:225-243 ISSN:1885-3137

Número 49 - Julio - 2017

\title{
Efectos de un entrenamiento pliométrico en extremidades superiores e inferiores en el rendimiento físico en jóvenes tenistas \\ Effects of upper and lower body plyometric training on physical performance in young tennis players
}

\author{
Elena Pardos-Mainer ${ }^{1,2}$, Oscar Ustero-Pérez ${ }^{1}$, Oliver Gonzalo-Skok $^{1}$
}

1.Facultad de Ciencias de la Salud. Universidad San Jorge, Zaragoza, España

2.GENUD (Growth, Exercise, Nutrition and Development) Research Group, Universidad de Zaragoza. España

\section{Resumen}

El objetivo del presente estudio fue evaluar el efecto de un entrenamiento pliométrico (ejercicios en extremidades superiores e inferiores) en acciones físicas explosivas específicas en jóvenes tenistas. Veintiún jóvenes tenistas (11 chicos, 10 chicas; media de edad $14.33 \pm 1.77$ años) participaron en el presente estudio y fueron divididos de forma no aleatoria en grupo control, el cual continuó con su entrenamiento habitual de preparación física y en experimental, al que se le sustituyó su entrenamiento de preparación física habitual por un programa de entrenamiento pliométrico dos veces por semana durante un período de 8 semanas. Las variables de rendimiento fueron analizadas a través de diferentes valoraciones: un sprint de 20 metros (tiempos parciales $5 \mathrm{~m}, 10 \mathrm{~m}$ y $15 \mathrm{~m}$ ), un sprint de $5+5$ metros con un cambio de dirección de $180^{\circ}$, un test de salto con contramovimiento bilateral (CMJ) y unilateral, un test de lanzamiento de balón medicinal a la máxima distancia (BM), un test de velocidad de servicio y un test de salto horizontal bilateral (SH) y unilateral (SHU). El efecto producido por el entrenamiento fue calculado en base al tamaño del efecto. Todas las variables de rendimiento fueron mejoradas en ambos grupos en comparación con los valores del pre-test. Además, el grupo experimental obtuvo mejoras sustanciales con respecto al grupo control en el SH, SHU, CMJ Y BM. Por lo tanto, el programa de entrenamiento pliométrico podría considerarse como un estímulo efectivo en la mejora de las acciones explosivas en jóvenes tenistas.

Palabras clave: Agilidad; fuerza explosiva; adolescentes; tenis

\section{Abstract}

The aim of the current study was to assess the effect of plyometric training (upper and lower body exercises) in explosive actions in young tennis players. Twenty-one young tennis players (11 males, 10 females, age $14.33 \pm 1.77$ years old) participated in the present study and were divided, in a non-randomized manner, into a control group which continued with a regular physical training and in a experimental group, which performed a biweekly plyometric training instead of the habitual fitness training during an 8-week period. Performance variables were assessed through several assessments; a 20-m sprint test (split $5 \mathrm{~m}, 10 \mathrm{~m}$ and $15 \mathrm{~m}$ ), a $5+5 \mathrm{~m}$ sprint with a $180^{\circ}$ change of direction test, a bilateral (CMJ) and unilateral countermouvement jump test, a maximum distance medicine ball throw test (BM), a speed serve test and a bilateral (SH) and unilateral (SHU) standing broad jump test. Performance's effect was calculated based on the effect size. All performance variables were improved in both group compared to the pre-test. Furthermore, substantial greater enhancements were obtained in $\mathrm{SH}, \mathrm{SHU}, \mathrm{CMJ}$ and $\mathrm{BM}$ in the experimental group in comparison to the control group. Therefore, the plyometric training program might be considered as an effective stimulus to improve explosive actions in young tennis players.

Key words: Agility; power; adolescent; tennis.

Facultad de Ciencias de la Salud. Universidad San Jorge, Zaragoza y GENUD (Growth, Exercise, Nutrition and Development) Research Group, Universidad de Zaragoza. España

Email: emainer13@gmail.com 
Pardos-Mainer, E.; Ustero-Pérez, O., y Gonzalo-Skok, O. (2017). Efectos de un entrenamiento pliométrico en extremidades superiores e inferiores en el rendimiento físico en jóvenes tenistas. RICYDE. Revista internacional de ciencias del deporte, 49(13), 225-243.

\section{Introducción}

$\mathrm{E}$ tenis es un deporte caracterizado por su dinámica intermitente, con esfuerzos a intervalos de moderada y alta intensidad, provocados por acciones repetitivas de corta duración, pero de gran intensidad (Kovacs, 2007). Los jugadores de tenis, para ser competitivos y alcanzar el éxito, necesitan combinar velocidad, agilidad y potencia con una alta capacidad aeróbica. Por lo tanto, se requiere de un alto nivel de desarrollo en cuatro habilidades fundamentales: táctica, técnica, física y psicológica para llegar a ser un jugador de alto rendimiento (Fernández-Fernández, Sanz-Rivas, y Mendez-Villanueva, 2014; Kovacs, 2007). Por otra parte, a la hora de diseñar programas de entrenamiento para los tenistas, es importante tener en cuenta los requerimiento físicos y fisiológico de los jugadores, ya que pueden variar según el nivel del jugador, el estilo de juego, el sexo o la superficie de la pista, entre otros (Fernández-Fernández y col., 2014).

En general, en los partidos de tenis disputados bajo las reglas oficiales de competición, los períodos de trabajo se sitúan en torno a los 5-10 s y los de descanso entre 10-20 s, respectivamente, a excepción de cuando se producen los cambios de pista (90-120 s) (TorresLuque, Sánchez-Pay, Fernández-García, y Palao, 2014). Además, la distancia media de un sprint durante el transcurso de un punto en el tenis es de 4 a $7 \mathrm{~m}$, con una media de cuatro cambios de dirección (Fernández-Fernández y col., 2014). El tiempo de reacción, la aceleración inicial y la agilidad juegan un papel fundamental donde los jugadores de tenis, deben ser capaces de reaccionar lo más rápido posible a las acciones realizadas por el oponente (Reid, Sibte, Clarke, y Whiteside, 2013). El saque es el golpe más importante desde un punto de vista estratégico, es por ello que junto a los movimientos cortos y explosivos alrededor de la pista, los tenistas están obligados a poseer un saque preciso y potente (Elliott, 2006). En relación a estos hechos, es importante recalcar la necesidad de que los tenistas posean un excepcional dinamismo en movimientos multidireccionales durante los partidos (Fernandez-Fernandez, Saez de Villarreal, Sanz-Rivas, y Moya, 2016). Por lo tanto, los programas de entrenamiento que mejoren estas habilidades serán de gran importancia para la optimización del rendimiento en los tenistas.

Durante los sprints o cambios de dirección se produce un corto período de tiempo de permanencia en el suelo ( $<100$ milisegundos), por lo que las habilidades comentadas anteriormente requieren de un alto desarrollo de energía y de la máxima potencia. (Brughelli, Cronin, Levin, y Chaouachi, 2008). Lloyd y col. observaron que el entrenamiento pliométrico muestra resultados positivos en las mejoras de este tipo de acciones, tanto en la pubertad como en los pre-púberes (Lloyd, Oliver, Hughes, y Williams, 2012). Además, la implantación de programas de entrenamiento pliométrico mejoran las acciones explosivas (capacidad de salto, agilidad y fuerza), así como el rendimiento específico en jóvenes futbolistas (RamirezCampillo y col., 2015; Ramirez-Campillo, Meylan, y col., 2014) y tenistas (Barber-Westin, Hermeto, y Noyes, 2010; Behringer, Neuerburg, Matthews, y Mester, 2013; FernandezFernandez, Ellenbecker, Sanz-Rivas, Ulbricht, y Ferrautia, 2013; Fernandez-Fernandez, Saez de Villarreal, y col., 2016; Fernandez-Fernandez, Sanz-Rivas, Kovacs, y Moya, 2015). También es interesante conocer que los efectos de los programas de entrenamiento pliométrico podrían ser diferentes dependiendo del momento de la maduración de los niños (Meylan, Cronin, Oliver, Hopkins, y Contreras, 2014) o del sexo en el que se llevaran a cabo (Haubenstricker y Seefeldt, 1986). 
Pardos-Mainer, E.; Ustero-Pérez, O., y Gonzalo-Skok, O. (2017). Efectos de un entrenamiento pliométrico en extremidades superiores e inferiores en el rendimiento físico en jóvenes tenistas. RICYDE. Revista internacional de ciencias del deporte, 49(13), 225-243.

https://doi.org/10.5232/ricyde2017.04903

En los último años, los jugadores de tenis dedican a un entrenamiento técnico y táctico un promedio de 15 a 20 horas semanales, incluso en edades tempranas, ya que la competitividad en el tenis se ha incrementado considerablemente (Reid, Crespo, Santilli, Miley, y Dimmock, 2007). De esta forma y debido a la escasez de tiempo, la inclusión de programas de entrenamiento que optimicen la mejora de las acciones explosivas en breves períodos de tiempo parecen ser recomendables. Por lo tanto, el objetivo del presente estudio fue evaluar el efecto de un entrenamiento pliométrico (extremidades superiores e inferiores) en acciones físicas explosivas específicas en jóvenes tenistas durante un período de ocho semanas.

\section{Método}

\section{Participantes}

En el presente estudio participaron voluntariamente veintiún jóvenes tenistas (edad: 14,33 \pm 1,77 años; altura: $170,13 \pm 9,49 \mathrm{~cm}$; peso: $62,63 \pm 11,71 \mathrm{~kg}$; índice de masa corporal: $21,47 \pm$ $2,6 \mathrm{~kg} / \mathrm{m} 2 ; 2$ zurdos y 19 diestros) divididos en 11 chicos y 10 chicas. La toma de datos serealizó en el $5^{\circ}$ mes de la temporada incluyendo la pre-temporada, estructurada en base a una planificación tradicional de un solo macrociclo debido a que son jugadores todavía en formación. El periodo de intervención, del $5^{\circ}$ al $7^{\circ}$ mes, pertenece al mesociclo específico de la planificación. Los jugadores se encontraban clasificados en los puestos más altos del Ránking Aragonés de Tenis (Top 20) en sus respectivas categorías (Infantil, Cadete y Júnior). Todos los sujetos habían jugado al tenis, como mínimo, durante los últimos cuatro años con una media aproximada de 12 horas de entrenamiento a la semana (5-6 sesiones/semana). Además durante el periodo que se llevó a cabo el estudio, compitieron en torneos autonómicos e internos del club, lo que supuso una carga de entrenamiento extra de 10 horas al mes. Los participantes estaban sanos y no habían sufrido ningún tipo de enfermedad ni lesión que pudiese afectar a los resultados en el momento del estudio. Cada participante entregó el correspondiente consentimiento informado cumplimentado por sus padres o tutores legales. El presente estudio fue aprobado por el Comité de Ética del Gobierno de Aragón (CEICA, España) y conforme a las recomendaciones de la Declaración de Helsinki.

\section{Técnicas instrumentales}

Las pruebas de campo se realizaron con un mínimo de 48 horas después de una competición o entrenamiento físico con el fin de minimizar la influencia de la fatiga. La evaluación del programa se realizó con una climatología y condiciones de superficie similares $\left(10-15^{\circ} \mathrm{C}\right.$, pista polvo de arcilla) de manera previa e inmediatamente después del período de ocho semanas de entrenamiento. Las pruebas se completaron en 1 hora siguiendo el mismo orden tanto en la primera (pre-test) como la segunda evaluación (post-test), incluyendo (a) composición corporal (b) salto con contramovimiento bilateral (CMJ) y unilateral (CMJU), (c) salto horizontal bilateral (SH) y unilateral (SHU), (d) lanzamiento de balón medicinal (BM), (e) sprint $20 \mathrm{~m}$, (f) cambio de dirección de $180^{\circ}$ (CD 180 $)$ y (g) velocidad de servicio (VS). Además, todas las mediciones fueron hechas antes del comienzo del entrenamiento, tras un calentamiento estandarizado de 10 minutos que incluía ejercicio aeróbico (5 min), movilización general (3 min) y ejercicios balísticos ( $2 \mathrm{~min}$ ).

Valoración de la composición corporal. La altura del cuerpo se midió con un estadiómetro portátil con una altura máxima de 2,10 m y un margen de error de $1 \mathrm{~mm}$ (SECA 225, SECA, Hamburgo, Alemania). La masa corporal fue medida con una balanza digital $( \pm 0,1 \mathrm{~kg}, \mathrm{ADE}$ Electronic Column Scales, Hamburgo, Alemania). Estas mediciones fueron realizadas siguiendo las directrices propuestas por la Sociedad Internacional para el Avance de la 
Pardos-Mainer, E.; Ustero-Pérez, O., y Gonzalo-Skok, O. (2017). Efectos de un entrenamiento pliométrico en extremidades superiores e inferiores en el rendimiento físico en jóvenes tenistas. RICYDE. Revista internacional de ciencias del deporte, 49(13), 225-243.

https://doi.org/10.5232/ricyde2017.04903

Cineantropometría (ISAK) (Eston, Eston, y Reilly, 2009). El Índice de Masa Corporal (IMC) fue calculado dividiendo el peso $(\mathrm{kg})$ por la altura al cuadrado $\left(\mathrm{m}^{2}\right)$.

Sprint $20 \mathrm{~m}$. Los jugadores realizaron un sprint de $20 \mathrm{~m}$ con tiempos parciales a los 5, 10 y 15 metros. Los sujetos se colocaban $0,5 \mathrm{~m}$ más atrás de la primera célula fotoeléctrica (Witty, Microgate, Bolzano, Italia). Cada jugador realizó dos sprints máximos intercalados con tres minutos de recuperación pasiva. El mejor tiempo fue utilizado para el posterior análisis estadístico.

Cambio de dirección de $180^{\circ}$ : En este test se realizó un desplazamiento que incluía un sprint de $10 \mathrm{~m}$. Los participantes iniciaban el test a una distancia de $0,5 \mathrm{~m}$ de la línea de salida/llegada, donde se colocaron las células fotoeléctricas (Witty, Microgate, Bolzano, Italia), cruzaban la línea de $5 \mathrm{~m}$ con el pie derecho o izquierdo, giraban $180^{\circ}$ y sprintaban de nuevo hacia la línea de salida/llegada (Gonzalo-Skok, Serna, Rhea, y Marin, 2015). Cada tenista realizó dos repeticiones con cada pie, intercaladas con tres minutos de recuperación pasiva, guardando el mejor tiempo para el posterior análisis estadístico. Las variables utilizadas en el análisis fueron $\mathrm{CD} 180^{\circ}$ con la pierna derecha (CDD) y con la pierna izquierda (CDI).

Test de salto con contramovimiento bilateral (CMJ). Este test fue realizado para medir la fuerza explosiva de las extremidades inferiores. Se utilizó un Optojump (MicroGate, Bolzano, Italia) para medir la altura de vuelo $(\mathrm{cm})$. Se instruyó a los participantes para poner las manos en sus caderas mientras realizaban un movimiento descendente seguido de un salto vertical máximo. Todos los sujetos fueron enseñados para caer en una posición vertical con las rodillas dobladas después del contacto con el suelo. Se realizaron tres saltos con un descanso pasivo de 45 segundos entre saltos (Fernandez-Fernandez, Saez de Villarreal, y col., 2016). El valor más alto de los tres intentos se utilizó para el posterior análisis estadístico.

Test de salto con contramovimiento unilateral (CMJU). En esta prueba, el tenista se encontraba en posición erguida con las manos detrás de la espalda, teniendo que efectuar un salto vertical máximo con una pierna después del contramovimiento hacia abajo (el grado de flexión fue autoseleccionado). Se utilizó un Optojump (MicroGate, Bolzano, Italia) para medir la altura de vuelo $(\mathrm{cm})$. Durante la acción de la flexión, el tronco debía permanecer lo más erguido posible para evitar cualquier posible influencia sobre el rendimiento de los miembros inferiores. Se permitió el balanceo de la pierna libre. El sujeto debía de caer sobre la misma pierna de impulso teniendo que permanecer sobre dicha pierna durante 2-3 segundos para considerar el intento como válido. Se realizaron dos repeticiones máximas, seleccionando el mejor intento para el posterior análisis estadístico. Se esperó un mínimo de 60 segundos entre cada repetición (Gonzalo-Skok y col., 2015). Las variables utilizadas para el análisis fueron CMJ con la pierna derecha (CMJUD) y CMJ con la pierna izquierda (CMJUI).

Test de lanzamiento de balón medicinal. El lanzamiento de balón medicinal por encima de la cabeza se realizó siguiendo el protocolo descrito por Ulbricht y col. (Ulbricht, Ferrauti, y Fernández-Fernández, 2013), utilizando un balón medicinal de $2 \mathrm{~kg}$. Para dicha medición se utilizó una cinta métrica estándar (30 m M13; Stanley, New Britain, EEUU). La distancia se midió en centímetros desde la línea de lanzamiento hasta el punto donde cayó el balón. Se realizaron dos lanzamientos, seleccionando el mejor intento para el posterior análisis estadístico. Se esperó un mínimo de 60 segundos entre cada repetición. 
Pardos-Mainer, E.; Ustero-Pérez, O., y Gonzalo-Skok, O. (2017). Efectos de un entrenamiento pliométrico en extremidades superiores e inferiores en el rendimiento físico en jóvenes tenistas. RICYDE. Revista internacional de ciencias del deporte, 49(13), 225-243.

https://doi.org/10.5232/ricyde2017.04903

Test de velocidad de servicio. En esta prueba se instruyó al jugador para realizar el saque con su técnica preferida, siempre y cuando cumpliera con unos métodos de técnica correctos (Fernandez-Fernandez y col., 2013). Para medir la velocidad de saque $(\mathrm{km} / \mathrm{h})$ se utilizó una pistola radar (Stalker Professional Sports Radar, Plymouth, MN, USA). El radar se coloca en el centro de la línea de fondo, cuatro metros por detrás del sacador, alineado con la altura aproximada de contacto con la pelota $(\sim 2.2 \mathrm{~m})$ y hacia el centro de la pista. Los jugadores realizaron ocho saques al máximo, con un máximo de 20 segundos de recuperación entre saque, todos en el lado del deuce, utilizando su propia raqueta y un conjunto de pelotas con las que realizaban su entrenamiento habitual. El número de repeticiones fue de ocho para tratar de disminuir el error producido por la desviación del saque con respecto a la posición del radar. Por ello, también se indicó a los jugadores de que debían de realizar el saque lo más centrado posible. Para que el saque contabilizase como válido, debía entrar en el cuadro de saque. La velocidad más alta registrada fue utilizada para el posterior análisis estadístico.

Test de salto horizontal bilateral. El salto horizontal bilateral fue realizado para medir la fuerza explosiva de las extremidades inferiores en la aplicación de fuerza horizontal. Para su medición se utilizó una cinta métrica estándar (30 m M13; Stanley, New Britain, EEUU). Los sujetos, de pie, con los pies colocados justo detrás de la línea (marcada con cinta) y con los brazos relajados, fueron instruidos para impulsarse y saltar la máxima distancia horizontal posible, ejecutando una caída controlada y mantener el equilibrio sobre ambas piernas (2-3 s) hasta que el evaluador registrase la posición de caída. La distancia se midió en centímetros desde la línea de batida hasta el talón más atrasado en la posición final (Rosch y col., 2000).Cada tenista realizó dos intentos con 60 segundos de recuperación entre saltos. El mejor intento fue seleccionado para el posterior análisis estadístico.

Test de salto horizontal unilateral. El sujeto comenzaba sobre un apoyo para, posteriormente, saltar con dicha pierna tratando de llegar lo más lejos posible y caer sobre la misma pierna de impulso. El balanceo de la pierna contraria fue permitido. Las manos debían colocarse entrelazadas detrás de la espalda. Los tenistas fueron instruidos para ejecutar una caída controlada y mantener el equilibrio sobre la pierna de caída (no se permitieron saltos extra) hasta que el evaluador registrase (2-3 s) la posición de caída. La distancia fue medida en centímetros desde la línea de batida hasta el talón en la posición final. Cada sujeto realizó dos intentos con cada pierna con 60 segundos de recuperación entre saltos (Gonzalo-Skok y col., 2015). El mejor intento fue seleccionado para el posterior análisis estadístico. Las variables utilizadas para el análisis fueron SH sobre pierna derecha (SHMD) y SH sobre pierna izquierda (SHMI).

\section{Procedimiento e intervención}

En el presente estudio se llevó a cabo un ensayo clínico no aleatorio. Los tenistas fueron divididos en cuatro grupos: Grupo Experimental Masculino (GEM, n=5), Grupo Experimental Femenino (GEF, n=6), Grupo Control Masculino $(\mathrm{GCM}, \mathrm{n}=6)$ y Grupo Control Femenino $(\mathrm{GCF}, \mathrm{n}=4)$. Todos estos grupos seguían la misma metodología de entrenamiento y un volumen de entrenamiento similar, con una media aproximada de 12 horas de entrenamiento a la semana (5-6 sesiones/semana). Todos los tenistas siguieron con su entrenamiento habitual en pista. Los grupos controles ( $\mathrm{GC}=\mathrm{GCM}$ y $\mathrm{GCF})$ continuaron con su entrenamiento habitual de preparación física (resistencia, velocidad, coordinación y agilidad). En cambio, los grupos experimentales (GE $=$ GEM y GEF) se sometieron a un programa de entrenamiento pliométrico (ejercicios de tren inferior y superior) dos veces por semana (días no consecutivos) durante ocho semanas. El entrenamiento de preparación física (45 min) fue llevado a cabo a continuación del entrenamiento en pista (90 min). Durante las dos semanas 
Pardos-Mainer, E.; Ustero-Pérez, O., y Gonzalo-Skok, O. (2017). Efectos de un entrenamiento pliométrico en extremidades superiores e inferiores en el rendimiento físico en jóvenes tenistas. RICYDE. Revista internacional de ciencias del deporte, 49(13), 225-243.

https://doi.org/10.5232/ricyde2017.04903

previas a la intervención, se realizaron cuatro sesiones de familiarización con los ejercicios a realizar, donde se realizaron correcciones de la técnica a través de indicaciones verbales y demostraciones por parte de los investigadores. La participación de los tenistas fue registrada durante el periodo de intervención.

Tabla 1. Datos descriptivos de los participantes/ Media \pm DS

\begin{tabular}{|c|c|c|c|c|c|c|c|}
\hline & $\begin{array}{l}\text { Sujetos } \\
\text { (n) }\end{array}$ & $\begin{array}{l}\text { Edad } \\
\text { (años) }\end{array}$ & $\begin{array}{l}\text { Altura } \\
(\mathrm{cm})\end{array}$ & $\begin{array}{r}\text { Peso } \\
(\mathrm{kg})\end{array}$ & $\begin{array}{c}\mathrm{IMC} \\
\left(\mathrm{kg} / \mathrm{m}^{2}\right)\end{array}$ & $\begin{array}{c}\text { Entrenamiento } \\
\text { semanal (h) }\end{array}$ & $\begin{array}{c}\text { Años } \\
\text { practicando } \\
\text { tenis }\end{array}$ \\
\hline $\begin{array}{c}\text { Grupo } \\
\text { Experimental } \\
\text { Masculino }\end{array}$ & 5 & $\begin{array}{r}16,26 \\
\pm 1,04\end{array}$ & $\begin{array}{l}181,44 \\
\pm 6,34\end{array}$ & $\begin{array}{c}70,9 \pm \\
5,23\end{array}$ & $\begin{array}{c}21,52 \pm \\
0,78\end{array}$ & 13 & $9 \pm 2$ \\
\hline $\begin{array}{c}\text { Grupo } \\
\text { Experimental } \\
\text { Femenino }\end{array}$ & 6 & $\begin{array}{l}12,57 \\
\pm 0,5\end{array}$ & $\begin{array}{l}161,83 \\
\pm 3,55\end{array}$ & $\begin{array}{c}53,58 \pm \\
10,91\end{array}$ & $\begin{array}{c}20,36 \pm \\
3,35\end{array}$ & 11 & $6 \pm 2$ \\
\hline $\begin{array}{l}\text { Grupo Control } \\
\text { Masculino }\end{array}$ & 6 & $\begin{array}{r}15,39 \\
\pm 0,92\end{array}$ & $\begin{array}{c}173,97 \\
\pm 2,4\end{array}$ & $\begin{array}{c}72,23 \pm \\
2\end{array}$ & $\begin{array}{c}23,88 \pm \\
1,01\end{array}$ & 13 & $8 \pm 2$ \\
\hline $\begin{array}{l}\text { Grupo Control } \\
\text { Femenino }\end{array}$ & 4 & $\begin{array}{c}13 \pm \\
0,7\end{array}$ & $\begin{array}{c}162,7 \pm \\
7,87\end{array}$ & $\begin{array}{c}51,45 \pm \\
6,62\end{array}$ & $\begin{array}{c}19,43 \pm \\
2,04\end{array}$ & 11 & $6 \pm 1$ \\
\hline
\end{tabular}

IMC: Índice de Masa Corporal

El programa de entrenamiento pliométrico consistió en una combinación de ejercicios del tren inferior y superior (Tabla 2). El número de ejercicios, series y repeticiones fue elegido en base a estudios anteriores realizados con jóvenes deportistas (Fernandez-Fernandez, Saez de Villarreal, y col., 2016; Ramirez-Campillo, Andrade, y Izquierdo, 2013; Ramirez-Campillo y col., 2015; Ramirez-Campillo, Meylan, y col., 2014). Así, un programa de 6-8 ejercicios, fue llevado a cabo a la máxima intensidad, con 2-4 series y 10-15 repeticiones. El periodo de descanso fue de dos minutos entre series y ejercicios. Se finalizaron todos los entrenamientos mediante un protocolo de enfriamiento de cinco minutos (movilización general y estiramientos estáticos pasivos). La presente intervención se llevó a cabo dos veces a la semana durante ocho semanas, es decir, fueron realizadas 16 sesiones. 
Pardos-Mainer, E.; Ustero-Pérez, O., y Gonzalo-Skok, O. (2017). Efectos de un entrenamiento pliométrico en extremidades superiores e inferiores en el rendimiento físico en jóvenes tenistas. RICYDE. Revista internacional de ciencias del deporte, 49(13), 225-243.

Tabla 2. Descripción del programa de entrenamiento pliométrico

\begin{tabular}{|c|c|c|c|c|c|c|}
\hline Sem & $\begin{array}{l}\text { Ejr } \\
(n)\end{array}$ & $\begin{array}{l}\mathrm{Sr} \\
(\mathrm{n})\end{array}$ & $\begin{array}{c}\text { Reps } \\
(\mathrm{n})\end{array}$ & $\begin{array}{c}\text { Des } \\
(\min )\end{array}$ & Ejercicios extremidades inferiores & Ejercicios extremidades superiores \\
\hline 1 & 6 & 2 & 15 & 2 & $\begin{array}{l}\text { Saltos de tobillo hacia delante 2-piernas; salto caja 2- } \\
\text { piernas; CMJ }\end{array}$ & $\begin{array}{l}\text { Pase de pecho; pase sobre cabeza; pase } \\
\text { posición cerrada }\end{array}$ \\
\hline 2 & 6 & 2 & 15 & 2 & CMJ; SM 2-piernas; saltos zig-zag sobre línea 2-piernas & $\begin{array}{l}\text { Pase sobre cabeza; pase posición abierta; } \\
\text { der/rev con mancuerna* }\end{array}$ \\
\hline 3 & 6 & 3 & 15 & 2 & $\begin{array}{l}\text { Saltos zig-zag sobre línea 2-piernas; SL con } \\
\text { estabilización; salto caja 1-pierna }\end{array}$ & $\begin{array}{l}\text { Golpe de balón medicinal sobre cabeza; } \\
\text { pase posición cerrada; der/rev con GE }\end{array}$ \\
\hline 4 & 6 & 3 & 15 & 2 & $\begin{array}{l}\text { CMJ; saltos multidireccionales 2/1-pierna; saltos zig- } \\
\text { zag sobre línea 2/1-pierna }\end{array}$ & $\begin{array}{l}\text { Pase de pecho; pase posición abierta; } \\
\text { der/rev con mancuerna* }\end{array}$ \\
\hline 5 & 8 & 4 & 12 & 2 & $\begin{array}{l}\text { SL de tobillo 2/1-pierna; SL con estabilización; salto } \\
\text { caja 2-piernas; CMJ }\end{array}$ & $\begin{array}{l}\text { Flexiones; pase sobre cabeza; pase } \\
\text { posición abierta; der/rev con GE }\end{array}$ \\
\hline 6 & 8 & 4 & 12 & 2 & $\begin{array}{c}\text { Saltos zig-zag sobre línea 2/1-pierna; SL con } \\
\text { estabilización; salto caja 2/1-piera; SL de tobillo 1- } \\
\text { pierna }\end{array}$ & $\begin{array}{c}\text { Pase de pecho; golpe de balón medicinal } \\
\text { sobre cabeza; der/rev con GE; der/rev con } \\
\text { mancuerna* }\end{array}$ \\
\hline 7 & 8 & 4 & 10 & 2 & $\begin{array}{l}\text { Salto de tobillo hacia delante } 2 / 1 \text { piernas; SL con } \\
\text { estabilización; } \mathrm{CMJ}\end{array}$ & $\begin{array}{l}\text { Flexiones (con palmada); pase de pecho; } \\
\text { pase posición cerrada/abierta; der/rev con } \\
\text { GE }\end{array}$ \\
\hline 8 & 8 & 4 & 10 & 2 & $\begin{array}{l}\text { CMJ; SM 2/1-pierna; saltos zig-zag sobre línea 2/1- } \\
\text { pierna; SL hacia delante de tobillo 2/1-pierna }\end{array}$ & $\begin{array}{l}\text { Pase sobre cabeza; flexiones (con } \\
\text { palmada); golpe de balón medicinal sobre } \\
\text { cabeza; der/rev con mancuerna* }\end{array}$ \\
\hline
\end{tabular}

Sem: semana; Ejr: ejercicios; Sr: series; reps: repeticiones; des: descanso; der: derecha; rev: revés; n: número; min: minutos; *: mancuerna 2 kg chicas y 3 kg chicos; ^: balón medicinal 2 kg chicas y $3 \mathrm{~kg}$ chicos; flexiones: chicas con rodillas y chicos con pies; CMJ: salto con contramovimiento ; SL: salto lateral; SM: saltos multidireccionales; GE: goma elástica 
Pardos-Mainer, E.; Ustero-Pérez, O., y Gonzalo-Skok, O. (2017). Efectos de un entrenamiento pliométrico en extremidades superiores e inferiores en el rendimiento físico en jóvenes tenistas. RICYDE. Revista internacional de ciencias del deporte, 49(13), 225-243.

https://doi.org/10.5232/ricyde2017.04903

\section{Análisis estadístico}

Los datos son presentados como media \pm desviación estándar (DE) y fueron analizados utilizando el Statistical Package for the Social Sciences 15,0 software (SPSS Inc.,USA). El estudio de las variables muestra una distribución normal de acuerdo a la prueba de Shapiro- Wilk. Se calculó el tamaño del efecto (TE, 90\% intervalo de confianza [IC]) en las variables seleccionadas, utilizando la DE del valor del pre-test ( $1^{\mathrm{a}}$ evaluación). Los valores cuantitativos para el tamaño del efecto de Cohen fueron (pequeño), 0,6 (moderado), y >1,2 (grande) (Hopkins, Marshall, Batterham, y Hanin.”, 2009). Para las comparaciones intra-/inter-grupo, se calcularon las probabilidades de que las diferencias en el rendimiento fueran mejores/mayores (es decir, mayor que el mínimo cambio apreciable [0,2 multiplicado por la DE entre sujetos, basado en el principio de la d de Cohen]), similares, o peores/menores. Las probabilidades cuantitativas de un efecto beneficial/mejor o perjudicial/peor se evaluaron cualitativamente de la siguiente manera: $<1 \%$, prácticamente imposible; $1 \%$ a $5 \%$, muy poco probable; $5 \%$ a $25 \%$, poco probable; $25 \%$ a $75 \%$, posible; $75 \%$ a $95 \%$, probable; $95 \%$ a $99 \%$, muy probable; y $>99 \%$, casi seguro (Hopkins y col., 2009). Si la probabilidad de tener un rendimiento beneficioso/mejor y perjudicial/peor fue para ambos $>5 \%$, el resultado se consideraba como no claro. De lo contrario, ese cambio se interpretó como la diferencia observada (Hopkins y col., 2009).

\section{Resultados}

Los cambios relativos y los resultados cualitativos del GCM y GEM se muestran en la tabla 3. El GCM y el GEM mejoraron sustancialmente el CDD, el CDI y la VS. Además el GEM también obtuvo mejoras sustanciales en el SHUD, el CMJ, el CMJUD, el sprint de $5 \mathrm{~m}$ y el BM.

Tabla 3. Cambios en las variables de rendimiento en el Grupo Control Masculino (GCM) y Grupo Experimental Masculino (GEM), Media \pm DE

\begin{tabular}{|c|c|c|c|c|c|c|}
\hline \multirow[b]{2}{*}{ Variable } & \multirow[b]{2}{*}{ Pre-test } & \multirow[b]{2}{*}{ Post-test } & \multicolumn{2}{|c|}{ Diferencia } & \multirow[b]{2}{*}{ Posibilidades $^{\delta}$} & \multirow[b]{2}{*}{ EC } \\
\hline & & & $\%(90 \%$ IC $)$ & Estandarizado (90 IC) ${ }^{\zeta}$ & & \\
\hline \multicolumn{7}{|c|}{ Test de salto horizontal $(\mathrm{cm})$} \\
\hline \multicolumn{7}{|l|}{ Bilateral } \\
\hline GCM & $202,67 \pm 30,09$ & $205,67 \pm 27,64$ & $1,7(-1,3 ; 4,9)$ & $0,09(-0,07 ; 0,25)$ & $11 / 88 / 1 \%$ & Poco probable \\
\hline GEM & $206,8 \pm 11,12$ & $212,6 \pm 6,02$ & $2,9(-2,3 ; 8,4)$ & $0,43(-0,35 ; 1,2)$ & $72 / 20 / 8 \%$ & No claro \\
\hline \multicolumn{7}{|c|}{ Unilateral derecha } \\
\hline GCM & $151,67 \pm 23,45$ & $152,83 \pm 22,71$ & $0,9(-4 ; 6)$ & $0,05(-0,22 ; 0,32)$ & $15 / 79 / 6 \%$ & No claro \\
\hline GEM & $149,8 \pm 10,5$ & $159,8 \pm 7,29$ & $6,8(-0,3 ; 14,4)$ & $0,76(-0,04 ; 1,55)$ & $90 / 7 / 3 \%$ & Probable \\
\hline \multicolumn{7}{|c|}{ Unilateral izquierda } \\
\hline GCM & $148,17 \pm 24,59$ & $153,17 \pm 20,07$ & $3,9(-0,8 ; 8,8)$ & $0,18(-0,04 ; 0,41)$ & $45 / 54 / 1 \%$ & Posible \\
\hline GEM & $153,4 \pm 6,11$ & $156,4 \pm 8,79$ & $1,9(-0,9 ; 4,7)$ & $0,38(-0,18 ; 0,94)$ & $74 / 22 / 5 \%$ & Posible \\
\hline \multicolumn{7}{|c|}{ Test de salto con contramovimiento bilateral $(\mathrm{cm})$} \\
\hline \multicolumn{7}{|l|}{ Bilateral } \\
\hline GCM & $30,4 \pm 7,62$ & $30,9 \pm 6,04$ & $2,9(-11,8 ; 20,2)$ & $0,07(-0,33 ; 0,47)$ & $26 / 64 / 10 \%$ & No claro \\
\hline GEM & $32,08 \pm 3,88$ & $35,72 \pm 4,21$ & $11,4(7,5 ; 15,4)$ & $0,67(0,45 ; 0,89)$ & $99 / 0 / 0 \%$ & Muy probable \\
\hline \multicolumn{7}{|c|}{ Unilateral derecha } \\
\hline GCM & $15,35 \pm 2,69$ & $15,65 \pm 2,72$ & $2,2(-12,4 ; 19,2)$ & $0,08(-0,51 ; 0,68)$ & $34 / 49 / 17 \%$ & No claro \\
\hline GEM & $15,86 \pm 3,97$ & $18,52 \pm 3,07$ & $18,2(8,7 ; 28,5)$ & $0,57(0,29 ; 0,86)$ & $98 / 2 / 0 \%$ & Muy probable \\
\hline
\end{tabular}


Pardos-Mainer, E.; Ustero-Pérez, O., y Gonzalo-Skok, O. (2017). Efectos de un entrenamiento pliométrico en extremidades superiores e inferiores en el rendimiento físico en jóvenes tenistas. RICYDE. Revista internacional de ciencias del deporte, 49(13), 225-243.

https://doi.org/10.5232/ricyde2017.04903

\begin{tabular}{|c|c|c|c|c|c|c|}
\hline GCM & $15,38 \pm 3,8$ & $15,43 \pm 2,17$ & $2,4(-17 ; 26,4)$ & $0,06(-0,47 ; 0,59)$ & $29 / 55 / 17 \%$ & No claro \\
\hline GEM & $18,18 \pm 3,37$ & $19,3 \pm 1,5$ & $7,3(-8,5 ; 25,9)$ & $0,31(-0,39 ; 1,02)$ & $63 / 28 / 10 \%$ & No claro \\
\hline \multicolumn{7}{|c|}{ Cambio de dirección de $180^{\circ}(\mathrm{s})$} \\
\hline \multicolumn{7}{|l|}{ Derecha } \\
\hline$\overline{\mathrm{GCM}}$ & $2,98 \pm 0,26$ & $2,83 \pm 0,25$ & $5(3 ; 6,9)$ & $0,43(0,26 ; 0,6)$ & $97 / 2 / 0 \%$ & Muy probable \\
\hline GEM & $2,85 \pm 0,06$ & $2,71 \pm 0,14$ & $5(0,4 ; 9,3)$ & $1,8(0,15 ; 3,46)$ & $95 / 2 / 3 \%$ & Muy probable \\
\hline \multicolumn{7}{|c|}{ Izquierda } \\
\hline GCM & $2,97 \pm 0,15$ & $2,81 \pm 0,27$ & $5,6(-1,1 ; 11,9)$ & $0,8(-0,16 ; 1,76)$ & $88 / 7 / 5 \%$ & Probable \\
\hline GEM & $2,85 \pm 0,07$ & $2,73 \pm 0,13$ & $4,3(1,1 ; 7,5)$ & $1,46(0,37 ; 2,55)$ & $97 / 2 / 2 \%$ & Muy probable \\
\hline \multicolumn{7}{|l|}{ Sprint $20 \mathrm{~m}(\mathrm{~s})$} \\
\hline \multicolumn{7}{|l|}{$5 \mathrm{~m}$} \\
\hline GCM & $1,12 \pm 0,06$ & $1,13 \pm 0,09$ & $-1,4(-5,5 ; 2,4)$ & $-0,19(-0,69 ; 0,32)$ & $9 / 44 / 48 \%$ & No claro \\
\hline GEM & $1,08 \pm 0,04$ & $1,04 \pm 0,06$ & $3,6(-0,8 ; 7,7)$ & $0,74(-0,15 ; 1,63)$ & $87 / 9 / 4 \%$ & Probable \\
\hline \multicolumn{7}{|l|}{$10 \mathrm{~m}$} \\
\hline GCM & $1,92 \pm 0,14$ & $1,91 \pm 0,17$ & $0,5(-2,6 ; 3,4)$ & $0,05(-0,26 ; 0,35)$ & $16 / 76 / 8 \%$ & No claro \\
\hline GEM & $1,84 \pm 0,06$ & $1,79 \pm 0,08$ & $1,5(-3,9 ; 6,7)$ & $0,35(-0,88 ; 1,58)$ & $61 / 21 / 18 \%$ & No claro \\
\hline \multicolumn{7}{|l|}{$15 \mathrm{~m}$} \\
\hline GCM & $2,64 \pm 0,21$ & $2,61 \pm 0,23$ & $1,2(-0,5 ; 2,8)$ & $0,11(-0,05 ; 0,27)$ & $14 / 85 / 1 \%$ & Poco probable \\
\hline GEM & $2,51 \pm 0,04$ & $2,48 \pm 0,09$ & $1,3(-1,8 ; 4,3)$ & $0,62(-0,82 ; 2,06)$ & $72 / 14 / 15 \%$ & No claro \\
\hline \multicolumn{7}{|l|}{$20 m$} \\
\hline GCM & $3,34 \pm 0,32$ & $3,31 \pm 0,31$ & $0,9(-0,6 ; 2,4)$ & $0,07(-0,05 ; 0,19)$ & $4 / 95 / 1 \%$ & Muy poco probable \\
\hline GEM & $3,16 \pm 0,06$ & $3,12 \pm 0,09$ & $1,3(-1,2 ; 3,9)$ & $0,57(-0,52 ; 1,67)$ & $75 / 15 / 10 \%$ & No claro \\
\hline \multicolumn{7}{|c|}{ Test de lanzamiento de balón medicinal $(\mathrm{cm})$} \\
\hline GCM & $861,67 \pm 76,27$ & $891,67 \pm 69,98$ & $3,6(-1,3 ; 8,6)$ & $0,33(-0,12 ; 0,77)$ & $70 / 27 / 3 \%$ & Posible \\
\hline GEM & $922 \pm 80,44$ & $1070 \pm 199,58$ & $15,8(5,9 ; 26,6)$ & $1,36(0,53 ; 2,18)$ & $98 / 1 / 1 \%$ & Muy probable \\
\hline \multicolumn{7}{|c|}{ Test de velocidad de saque $(\mathrm{km} / \mathrm{h})$} \\
\hline GCM & $134,33 \pm 18,22$ & $140,5 \pm 17,38$ & $4,7(2,1 ; 7,4)$ & $0,27(0,12 ; 0,42)$ & $82 / 18 / 0 \%$ & Probable \\
\hline GEM & $156,34 \pm 10,59$ & $161,6 \pm 7,02$ & $3,5(0,4 ; 6,7)$ & $0,4(0,05 ; 0,75)$ & $85 / 14 / 1 \%$ & Probable \\
\hline
\end{tabular}

GCM: Grupo control masculino; GEM: Grupo experimental masculino; DE: desviación estándar; EC: evaluación cualitativa; IC: Intervalo de confianza; cm: centímetros; s: segundos; Km/h: kilómetros/hora; $\delta$ : Tamaño de efecto; ${ }^{\zeta}$ : Porcentaje de cambio de mejores/ similares/ peores valores. Nota: Para mayor claridad, todas diferencias son presentadas como mejoras (positivas), así que diferencias negativas y positivas están en la misma dirección.

Los resultado del análisis intergrupo masculino están ilustrados en la figura 1. Se observó una mejora sustancial del GEM respecto a GCM en el SHUD (5,9\% [90IC: -2; 14,3] con cambios para mejores/similar/peores valores de 75/22/4\% respectivamente), el CMJUD (15,7\% [90IC: -0,8; 34,9], 86/11/2\%), el 5m (5\% [90IC: -0,1; 9,7], 90/7/3\%)y el BM (11,8\% [90IC: 1,9; 22,8], 94/4/2\%). No hubo diferencias sustanciales entre los grupos en el resto de variables. 
Pardos-Mainer, E.; Ustero-Pérez, O., y Gonzalo-Skok, O. (2017). Efectos de un entrenamiento pliométrico en extremidades superiores e inferiores en el rendimiento físico en jóvenes tenistas. RICYDE. Revista internacional de ciencias del deporte, 49(13), 225-243.

\section{Grupo experimental comparado grupo control masculino}

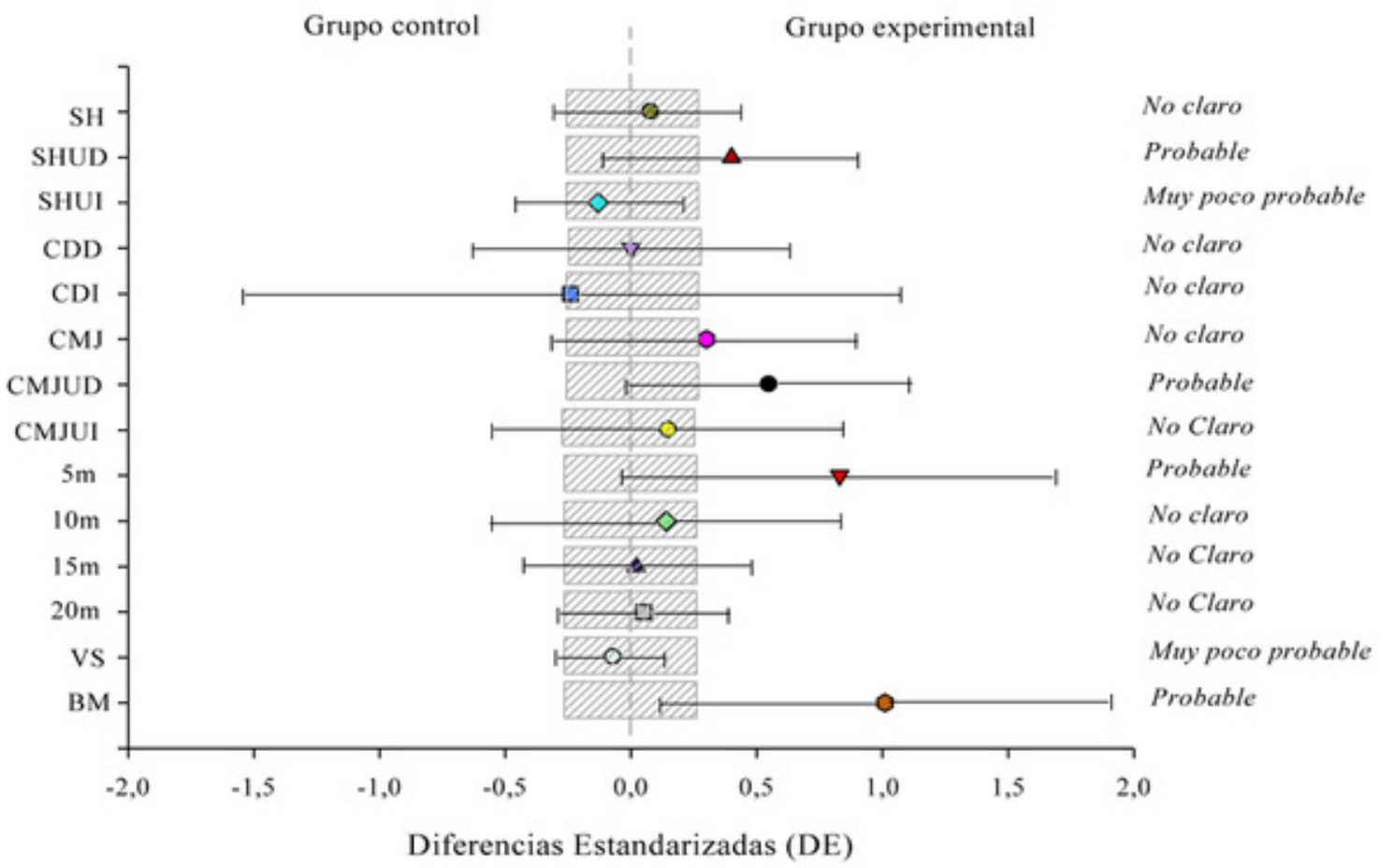

SH: Salto Horizontal; SHUD: Salto Horizontal Unilateral Derecha; SHUI: Salto Horizontal Unilateral Izquierda; CDD: Cambio dirección derecha; CDI: Cambio dirección izquierda; CMJ: Salto con contramovimiento: CMJUD: Salto con contramovimiento unilateral derecha; CMJUI: Salto con contramovimiento unilateral izquierda; BM: Lanzamiento balón medicinal sobre cabeza; VS: Velocidad del servicio; DE: Desviación estándar;

Figura 1. Eficiencia del grupo experimental masculino comparado con el grupo control masculino para mejorar las variables de fuerza, agilidad y velocidad. Las barras indican la incertidumbre presente en los verdaderos cambios en la media al $90 \%$ de los intervalos de confianza. Las áreas triviales fueron calculadas sobre el mínima cambio apreciable (mirar apartado de métodos).

Por otro lado, los cambios relativos y los resultados cualitativos del GCF y GEF se muestran en la tabla 4. El GCF y el GEF mejoraron sustancialmente CMJUD. Además el GEM también mejoró SHUD, SHUI, CDI, CMJ, CMJUI, BM y VS. 
Pardos-Mainer, E.; Ustero-Pérez, O., y Gonzalo-Skok, O. (2017). Efectos de un entrenamiento pliométrico en extremidades superiores e inferiores en el rendimiento físico en jóvenes tenistas. RICYDE. Revista internacional de ciencias del deporte, 49(13), 225-243.

https://doi.org/10.5232/ricyde2017.04903

Tabla 4. Cambios en las variables de rendimiento en el Grupo Control Femenino (GCF) y el Grupo Experimental Femenino (GEF), Media \pm DE

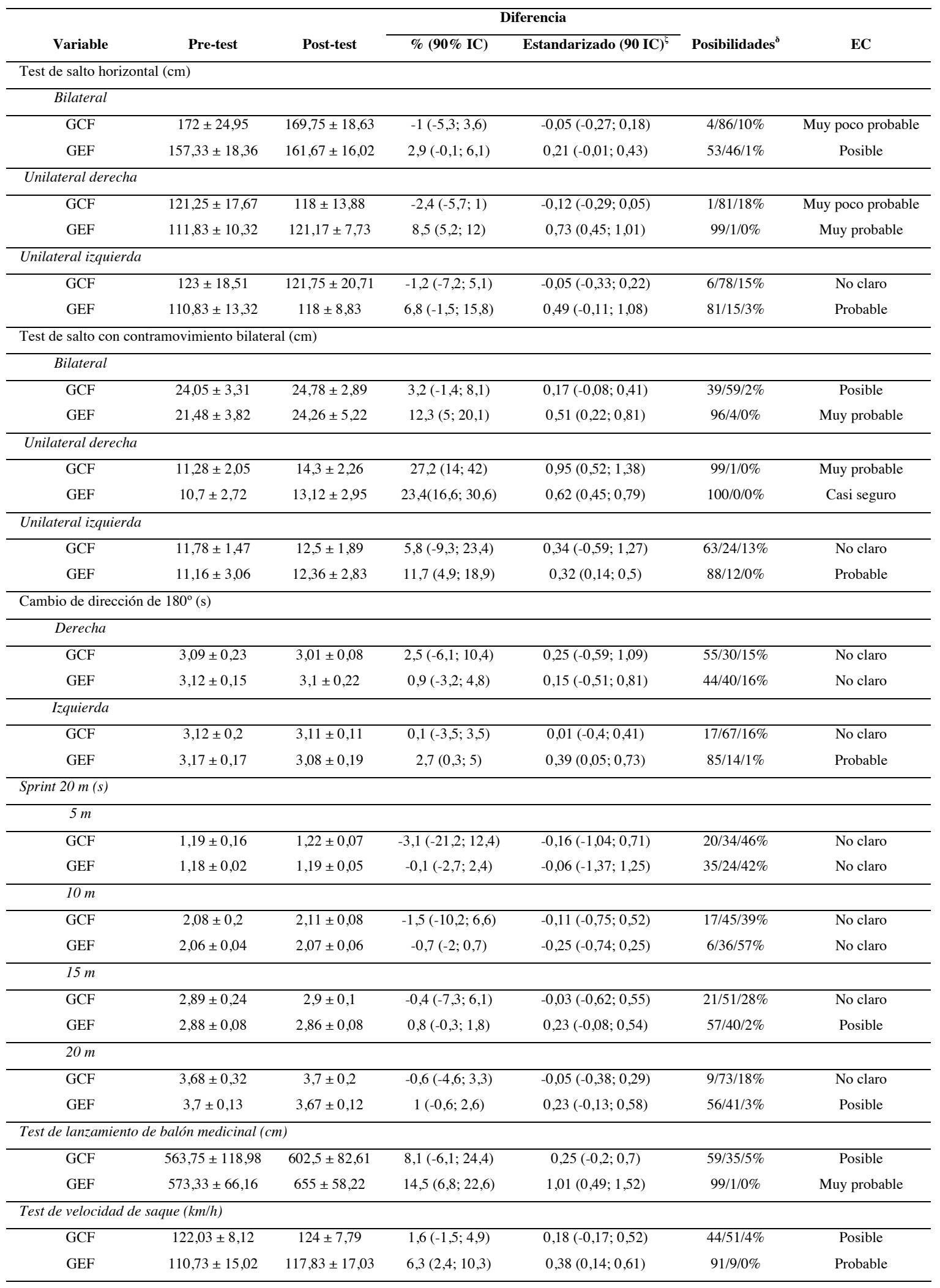

GCM: Grupo control masculino; GEM: Grupo experimental masculino; DE: desviación estándar; EC: evaluación cualitativa; IC: Intervalo de confianza; cm: centímetros; s: segundos; Km/h: kilómetros/hora; ${ }^{\delta}$ : Tamaño de efecto; ${ }^{\zeta}$ : Porcentaje de cambio de mejores/ similares/ peores valores. Nota: Para mayor claridad, todas diferencias son presentadas como mejoras (positivas), así que diferencias negativas y positivas están en la misma dirección. 
Pardos-Mainer, E.; Ustero-Pérez, O., y Gonzalo-Skok, O. (2017). Efectos de un entrenamiento pliométrico en extremidades superiores e inferiores en el rendimiento físico en jóvenes tenistas. RICYDE. Revista internacional de ciencias del deporte, 49(13), 225-243.

https://doi.org/10.5232/ricyde2017.04903

Los resultado del análisis intergrupo femenino están ilustrados en la figura 2. Se observó en el SH (3,9\% [90IC: 0,9; 8,9], 60/39/1\%), SHUD (11,2\% [90IC: 6,8; 15,8], 100/0/0\%), el SHUI $(8,1 \%$ [90IC: $-1,3 ; 18,4], 80 / 17 / 3 \%)$, el CDI $(2,6 \%$ [90IC: $-1,1$; 6,2], 73/23/4\%), el CMJ (8,8\% [90IC: 1,$2 ; 16,9], 55 / 15 / 1 \%)$ y el VS (4,6\% [90IC: 0,1 ; $9,2], 73 / 27 / 1 \%$ ) una mejora sustancialmente mayor del GEF respecto al GCF. No hubo diferencias sustanciales entre los grupos en el resto de variables.

\section{Grupo experimental comparado grupo control femenino}

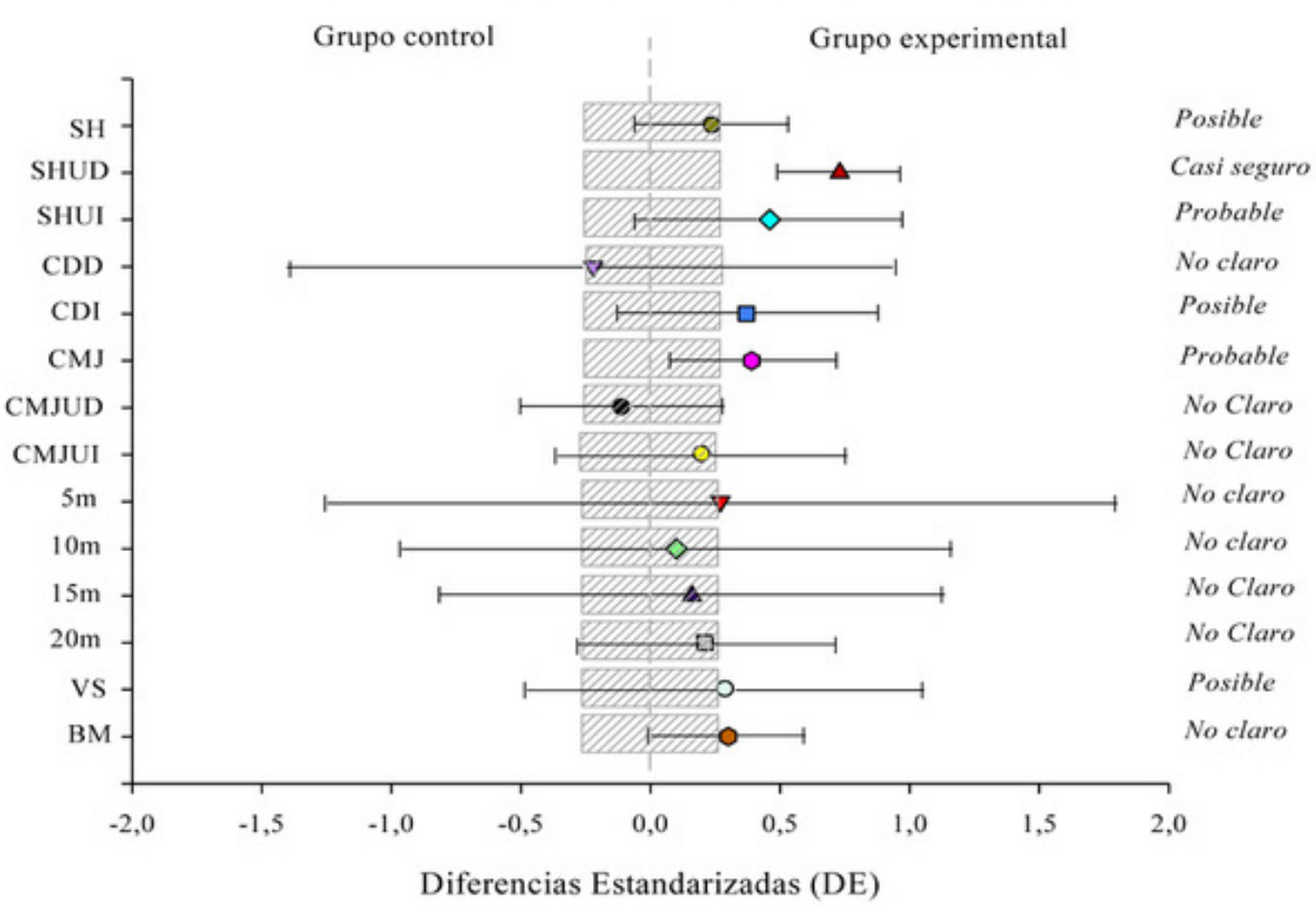

SH: Salto Horizontal; SHUD: Salto Horizontal Unilateral Derecha; SHUI: Salto Horizontal Unilateral Izquierda; CDD: Cambio dirección derecha; CDI: Cambio dirección izquierda; CMJ: Salto con contramovimiento: CMJUD: Salto con contramovimiento unilateral derecha; CMJUI: Salto con contramovimiento unilateral izquierda; BM: Lanzamiento balón medicinal sobre cabeza; VS: Velocidad del servicio; DE: Desviación estándar; m: metros

Figura 2. Eficiencia del grupo experimental femenino comparado con el grupo control femenino para mejorar las variables de fuerza, agilidad y velocidad. Las barras indican la incertidumbre presente en los verdaderos cambios en la media al $90 \%$ de los intervalos de confianza. Las áreas triviales fueron calculadas sobre el mínima cambio apreciable (mirar apartado de métodos).

\section{Discusión}

El objetivo del presente estudio fue evaluar el efecto de un entrenamiento pliométrico (extremidades inferiores y superiores), tras 8 semanas, en acciones físicas explosivas específicas en jóvenes tenistas. Nuestros resultados mostraron que un entrenamiento pliométrico parece ser un estímulo adecuado para la mejora de las cualidades físicas de los jóvenes jugadores de tenis. Por otro lado, no se observaron grandes cambios en las variables de las pruebas en el GC, lo que demuestra la importancia de la formación específica para poder mejorar las acciones explosivas de los jugadores de tenis. Además, las variables de CD mejoraron sustancialmente en los dos grupos (GC y GE), por lo que esta variable podría estar más asociada al entrenamiento en pista que al propio programa de entrenamiento pliométrico. 
Pardos-Mainer, E.; Ustero-Pérez, O., y Gonzalo-Skok, O. (2017). Efectos de un entrenamiento pliométrico en extremidades superiores e inferiores en el rendimiento físico en jóvenes tenistas. RICYDE. Revista internacional de ciencias del deporte, 49(13), 225-243.

https://doi.org/10.5232/ricyde2017.04903

La fuerza y potencia muscular en extremidades tanto superiores como inferiores son importantes con el fin de producir acciones explosivas en el tenis (el movimiento del saque, aceleraciones, cambios de dirección) (Girard y Millet, 2009). Existen Pocos estudios que hayan analizado el efecto de un programa de entrenamiento pliométrico sobre las cualidades físicas en jugadores de tenis (Fernandez-Fernandez, Saez de Villarreal, y col., 2016; Fernandez-Fernandez y col., 2015). En relación a los cambios observados en el CMJ en el GEM (TE: 0,43), la magnitud fue similar al observado en estudios previos con jugadores de tenis de 12-13 años (TE: 0,46) (Fernandez-Fernandez, Saez de Villarreal, y col., 2016) y mayor que en tenistas de 16-17 años (TE: 0,27) (Fernandez-Fernandez y col., 2015). Teniendo en cuenta que nuestros sujetos del GEM tienen 16-17 años, el cambio es mayor que el observado en el estudio de FernándezFernández y col. (Fernandez-Fernandez y col., 2015) el cual combinaba un trabajo de fuerza explosiva y sprints repetidos. Es posible que esta diferencia pueda ser debida a una mayor densidad de saltos verticales en nuestro programa pliométrico. En el GEF el cambio de magnitud del CMJ (ES: 0,51) es muy similar al observado en el estudio de Fernández- Fernández y col. (Fernandez-Fernandez y col., 2015) por lo que este tipo de entrenamiento pliométrico con la misma frecuencia y duración podría tener la misma repercusión en tenistas de misma edad, pero de diferente sexo. Además, comparando la pierna izquierda con la derecha en el CMJU, se observó, tanto en chicos como en chicas, una mayor mejora en la pierna derecha (TE: 0,57 y 0,62) con respecto a la pierna izquierda (TE: 0,31 y 0,31). Estos resultados no están en consonancia con la lógica del saque, en el cual existe una mayor implicación de la pierna no dominante, debido a la carga excéntrica que soporta el pie de caída durante el saque (Murphy, Duffield, Kellett, y Reid, 2015). Observando nuestros datos, podemos ver que en la primera evaluación los tenistas saltaban más con la pierna no dominante que la dominante, pero tras la intervención las mejoras fueron superiores con la pierna dominante. Por lo tanto, al inicio nuestros datos estaban en consonancia con los de Murphy y col. (Murphy y col., 2015), pero, tras la intervención, cambiaron probablemente debido a la utilización de ejercicios específicos unilaterales en los que el efecto tienda a ser más rápido en la pierna dominante.

Con respecto al SH, Markovic y Mikulic (Markovic y Mikulic, 2010) establecen que un programa de entrenamiento pliométrico podría aumentar el rendimiento en el salto horizontal. Fernández- Fernández y col. (Fernandez-Fernandez, Saez de Villarreal, y col., 2016) observaron mejoras en el SH (TE: 1,08) en tenistas de 17 años, superiores a las observadas en el GEM (TE: 0,43). Esta diferencia puede ser debida a la edad. Nuestros datos muestran mejores resultados del $\mathrm{CMJ}$ respecto al $\mathrm{SH}$ tanto en chicos (TE: 0,67 y 0,43 ) como en chicas (TE: 0,51 y 0,21 ), lo cual podría ser explicado por tener una combinación mayor de saltos verticales con respecto a los saltos laterales y horizontales, en contraste con el estudio previamente comentado (Fernandez-Fernandez, Saez de Villarreal, y col., 2016). Respecto a las diferencias entre pierna derecha e izquierda en el SHU en chicos y chicas, se observó un tamaño del efecto moderado de la pierna derecha (TE: 0,76 y 0,73) en comparación con un tamaño de efecto pequeño de la pierna izquierda (TE: 0,38 y 0,49 ), coincidiendo con los resultados expuestos en el párrafo anterior del salto vertical unilateral.

Se han destacado los programas de entrenamiento pliométrico como uno de los métodos de entrenamiento más útiles para la mejora en la velocidad de jóvenes deportistas (1115 años en chicas y 12-16 años en chicos) (Rumpf y col., 2015). En el test de sprint 
Pardos-Mainer, E.; Ustero-Pérez, O., y Gonzalo-Skok, O. (2017). Efectos de un entrenamiento pliométrico en extremidades superiores e inferiores en el rendimiento físico en jóvenes tenistas. RICYDE. Revista internacional de ciencias del deporte, 49(13), 225-243.

https://doi.org/10.5232/ricyde2017.04903

lineal de 20 metros con parciales cada 5 metros, se observó que el GEM (TE: 0,57) mejoró más respecto al GEF (TE: 0,23). Además, en todos los tiempos parciales, el GEM mostró una reducción considerable respecto al GCM de baja a moderada (TE: $0,35$ a 0,74$)$, mientras que el GEF mejoró ligeramente sus tiempos respecto al GCF, sin estar claro este cambio de magnitud. En diferentes estudios, se ha observado una reducción significativa del tiempo y el tamaño de efecto en los parciales de los test de velocidad de 20 metros (TE: 0,73 a 0,97) (Fernandez-Fernandez, Saez de Villarreal, y col., 2016) y 30 metros (TE: 0,74 a 1,08) (Fernandez-Fernandez y col., 2015) tras un programa de entrenamiento pliométrico. Estos resultados se asemejan parcialmente con los obtenidos por el GEM aunque el cambio es notablemente mayor en el estudio de Fernández- Fernández y col. (Fernandez-Fernandez, Saez de Villarreal, y col., 2016). Observando los datos de este estudio, los tiempos de los tenistas en esta prueba son mayores que los de nuestros sujetos por lo que sería más fácil tras la intervención mejorar sus tiempos respecto a los nuestros y, es por ello, por lo que quizás exista esta diferencia. Únicamente, se observó una mejora sustancial en el tiempo en $5 \mathrm{~m}$ en el grupo de tenistas masculino (TE: 0,74). Este hecho podría estar relacionado con los ejercicios específicos de entrenamiento pliométrico llevados a cabo, ya que la combinación de saltos multidireccionales que incluyen desplazamientos horizontales podría mejorar la aceleración y la propulsión horizontal (Ramirez-Campillo y col., 2015). En lo que respecta a las chicas, el cambio de magnitud en este test no está claro, siendo en los parciales de 15 y 20 metros donde se observó una pequeña mejora (TE: $0,23)$, similar a otros estudios que realizaron un programa de entrenamiento pliométrico (6-8 semanas, 1-2 días/semana) en corredoras (TE: 0,3) (Ramirez-Campillo, Alvarez, y col., 2014) y futbolistas (TE: 0,22) (Ozbar, Ates, y Agopyan, 2014).

Con respecto a la agilidad, el test CD $180^{\circ}$ (2 a 4 s) se asemeja más a la intensidad, duración y movimientos típicos en el entrenamiento y los partidos de tenis (Murphy y col., 2015). El entrenamiento pliométrico, no es sólo utilizado en los tenistas para entrenar la fuerza y los movimientos explosivos, sino también para mejorar la habilidad de desacelerar rápidamente de manera segura (Kovacs, Roeter, y Ellenbecker, 2008). Algunos estudios han observado mejoras en la agilidad de los tenistas tras diferentes programas de entrenamiento durante 6-9 semanas (Barber-Westin y col., 2010; Fernandez-Fernandez, Saez de Villarreal, y col., 2016; Fernandez-Fernandez, Sanz, Sarabia, y Moya, 2016; Salonikidis y Zafeiridis, 2008; Sannicandro, Cofano, Rosa, y Piccinno, 2014). No obstante, alguno de ellos utilizan test de agilidad con grandes diferencias (mayor o menor duración (2 a $60 \mathrm{~s}$ ), distancias más o menos largas (4 a 46,6m) o uso de raqueta) (Barber-Westin y col., 2010; Salonikidis y Zafeiridis, 2008; Sannicandro y col., 2014), lo que no permite compararlos con nuestro estudio. En el presente estudio, se diferenció entre pierna pivotante derecha (CDD) y pierna pivotante izquierda (CDI). Los chicos mejoraron sustancialmente el CDD (TE: 1,8) y CDI (TE: 1,46), mientras que en las chicas esta mejora no fue tan clara CDD (TE: 0,15) y CDI (TE: 0,39). No obstante, esta mejora es probable que no haya sido por el entrenamiento pliométrico, dado que en el análisis inter-grupo solo se hallaron diferencias en el CDI, ya que tanto grupo control como experimental mejoraron en la segunda evaluación con ambas piernas. Este hecho podría ser debido a que el entrenamiento realizado en pista implica acciones excéntricas constantes del tren inferior (aceleraciones y frenadas), aspecto fundamental para la capacidad de cambiar de dirección. 
Pardos-Mainer, E.; Ustero-Pérez, O., y Gonzalo-Skok, O. (2017). Efectos de un entrenamiento pliométrico en extremidades superiores e inferiores en el rendimiento físico en jóvenes tenistas. RICYDE. Revista internacional de ciencias del deporte, 49(13), 225-243.

https://doi.org/10.5232/ricyde2017.04903

La rotación del tronco que se produce durante el saque o los golpes en pista, principalmente aquellos que se realizan en una posición más abierta, requieren de estabilidad abdominal y fuerza (Knudson y Blackwell, 2000). La combinación de la rotación de tronco y coordinación de extremidades superiores (rotación interna de hombro, extensión de codo y flexión de muñeca) son componentes imprescindibles que ayudan a conseguir una óptima velocidad de golpeo y una posición correcta a la hora de golpear la pelota (Lees, 2003). El saque es un golpe importante en el tenista (Elliott, 2006), y el aumento de su velocidad media parece ser un objetivo claro de entrenamiento en la planificación de los programas de acondicionamiento físico en los jóvenes jugadores de tenis (Behringer y col., 2013). Con respecto a los test de velocidad de servicio, fuerza y resistencia de abdomen y extremidades superiores (lanzamiento de balón medicinal, 10 repeticiones máximas de press de pierna, pecho, abdomen), diferentes estudios han mostrado mejoras significativas tras un programa de entrenamiento en extremidades superiores e inferiores (Barber-Westin y col., 2010; Behringer y col., 2013; Fernandez-Fernandez y col., 2013; Fernandez-Fernandez, Saez de Villarreal, y col., 2016). En el presente estudio, también se hallaron mejoras sustanciales en el GEM y GEF tanto en la prueba de la VS (TE: 0,4 y 0,38) como en el BM (TE: 1,36 y 1,01). Además, al comparar en ambos sexos el GE con el GC, se observó que el GE mejoró más que el GC, pero esta mejora fue mucho menor en el test de la VS que en el BM. Esta diferencia puede ser debida a una falta de transferencia de la fuerza al gesto específico (saque), por lo que, quizás, en el programa de entrenamiento pliométrico haría falta un mayor número de ejercicios específicos para que la mejora de la VS se acercase a la del BM. A pesar de ello, el entrenamiento pliométrico parece ser considerado como un buen método para lograr una mayor coordinación intermuscular mejorando así la transferencia de la fuerza, a través de la cadena cinética, para una mejora concomitante del saque (Fernandez-Fernandez, Saez de Villarreal, y col., 2016). Estudios previos han observado mejoras significativas en la VS (ES: 0,79; ES: 0,41) y el BM (ES: 0,52) (Fernandez-Fernandez y col., 2013; Fernandez-Fernandez, Saez de Villarreal, y col., 2016). Dichos resultados están en consonancia con los nuestros y esto puede ser debido a que algunos ejercicios del programa de entrenamiento coinciden en los diferentes estudios y podrían repercutir positivamente en el rendimiento de los sujetos.

Tras analizar todos los datos resultantes de los test de velocidad y agilidad del presente estudio, se observó que las chicas obtienen peores resultados que los chicos tras un programa de entrenamiento pliométrico, por lo que quizás en futuros estudios se podría llevar a cabo un entrenamiento combinado (fuerza explosiva de extremidades inferiores + sprints repetidos) y observar las posibles mejoras en el rendimiento.

En cuanto a las limitaciones del estudio, cabe mencionar que la muestra utilizada es pequeña y los datos se limitan a un determinado grupo de tenistas, por lo que sería interesante realizar más estudios para confirmar los presentes resultados. Los jugadores de tenis adolescentes tiene unas características particulares que no permiten extrapolar nuestros resultados a otros deportes de manera directa. Estudios futuros deben extender estas observaciones a otros grupos de edad, niveles competitivos y muestras mayores con el fin de analizar si los resultados son similares. También sería interesante observar diferentes intensidades y volúmenes en el programa de entrenamiento pliométrico, con el fin de determinar la dosis óptima para este método de entrenamiento, así como observar si dicho programa puede reducir la incidencia de lesiones en jóvenes tenista. 
Pardos-Mainer, E.; Ustero-Pérez, O., y Gonzalo-Skok, O. (2017). Efectos de un entrenamiento pliométrico en extremidades superiores e inferiores en el rendimiento físico en jóvenes tenistas. RICYDE. Revista internacional de ciencias del deporte, 49(13), 225-243.

\section{Conclusión}

El presente estudio sugiere que la implantación de un programa de entrenamiento pliométrico, compuesto por 16 sesiones, en sustitución de un entrenamiento habitual de preparación física (resistencia, velocidad, coordinación y agilidad), parece ser un estímulo adecuado para la mejora de las cualidades físicas en jóvenes tenistas, tanto en chicos como en chicas. A este respecto, la participación en un programa de entrenamiento pliométrico, ha permitido que los jóvenes tenistas mejoren su fuerza explosiva y potencia muscular de extremidades superiores e inferiores, tendiendo a mejorar la pierna dominante respecto a la no dominante debido a la utilización de ejercicios específicos unilaterales no estando en consonancia con la lógica del saque (Murphy y col., 2015). Por otra parte, las variables de CD mejoraron sustancialmente en los dos grupos (GC y GE), por lo que esta variable podría estar más asociada al entrenamiento en pista que al propio programa de entrenamiento pliométrico. Por ello, aquellos entrenadores y preparadores físicos de tenis que quieran mejorar la condición física de sus jugadores deberían de incluir programas de entrenamiento que incluyeran ejercicios pliométricos para optimizar su rendimiento físico.

\section{Referencias}

Barber-Westin, S. D.; Hermeto, A. A., \& Noyes, F. R. (2010). A six-week neuromuscular training program for competitive junior tennis players. Journal of strength and conditioning research / National Strength \& Conditioning Association, 24(9), 2372-2382. https://doi.org/10.1519/JSC.0b013e3181e8a47f

Behringer, M.; Neuerburg, S.; Matthews, M., \& Mester, J. (2013). Effects of two different resistance-training programs on mean tennis-serve velocity in adolescents. Pediatric Exercise Sciences, 25(3), 370-384.

https://doi.org/10.1123/pes.25.3.370

Brughelli, M.; Cronin, J.; Levin, G., \& Chaouachi, A. (2008). Understanding change of direction ability in sport: a review of resistance training studies. Sports medicine, 38(12), 1045-1063. https://doi.org/10.2165/00007256-200838120-00007

Elliott, B. (2006). Biomechanics and tennis. British journal of sports medicine, 40(5), 392-396. https://doi.org/10.1136/bjsm.2005.023150

Eston, R.; Eston, R. G., \& Reilly, T. (2009). Kinanthropometry and Exercise Physiology Laboratory Manual: Anthropometry. London: UK: Taylor \& Francis. https://doi.org/10.1123/pes.2015-0019

Fernandez-Fernandez, J.; Ellenbecker, T.; Sanz-Rivas, D.; Ulbricht, A., \& Ferrautia, A. (2013). Effects of a 6-week junior tennis conditioning program on service velocity. Journal of sports science \& medicine, 12(2), 232-239.

Fernandez-Fernandez, J.; Saez de Villarreal, E.; Sanz-Rivas, D., \& Moya, M. (2016). The Effects of 8-Week Plyometric Training on Physical Performance in Young Tennis Players. Pediatric Exercise Science, 28(1), 77-86.

https://doi.org/10.1123/pes.2015-0019 
Pardos-Mainer, E.; Ustero-Pérez, O., y Gonzalo-Skok, O. (2017). Efectos de un entrenamiento pliométrico en extremidades superiores e inferiores en el rendimiento físico en jóvenes tenistas. RICYDE. Revista internacional de ciencias del deporte, 49(13), 225-243.

https://doi.org/10.5232/ricyde2017.04903

Fernandez-Fernandez, J.; Sanz, D.; Sarabia, J. M., \& Moya, M. (2016). The Effects of Sport-Specific Drills Training or High-Intensity Interval Training in Young Tennis Players. International journal of sports physiology and performance, 123.

Fernandez-Fernandez, J.; Sanz-Rivas, D.; Kovacs, M. S., \& Moya, M. (2015). Inseason effect of a combined repeated sprint and explosive strength training program on elite junior tennis players. Journal of strength and conditioning research / National Strength \& Conditioning Association, 29(2), 351-357. https://doi.org/10.1519/JSC.0000000000000759

Fernández-Fernández, J.; Sanz-Rivas, D., \& Mendez-Villanueva, A. (2014). A Review of the Activity Profile and Physiological Demands of Tennis Match Play. Strength and Conditioning Journal, 31(4), 15-26. https://doi.org/10.1519/SSC.0b013e3181ada1cb

Girard, O., \& Millet, G. P. (2009). Physical determinants of tennis performance in competitive teenage players. Journal of strength and conditioning research / National Strength \& Conditioning Association, 23(6), 1867-1872. https://doi.org/10.1519/JSC.0b013e3181b3df89

Gonzalo-Skok, O.; Serna, J.; Rhea, M. R., \& Marin, P. J. (2015). Relationships between Functional Movement Tests and Performance Tests in Young Elite Male Basketball Players. International journal of sports physical therapy, 10(5), 628638.

Haubenstricker, J., \& Seefeldt, V. (1986). Acquisition of motor skills during childhood. In Seefeldt (ed.), Physical activity and well-being: American Alliance for Health, Physical Education, Recreation and Dance.

Hopkins, W. G.; Marshall, S. W.; Batterham, A. M., \& Hanin, J. (2009). Progressive statistics for studies in sports medicine and exercise science. Medicine and science in sports and exercise, 41(1), 3-13. https://doi.org/10.1249/MSS.0b013e31818cb278

Knudson, D., \& Blackwell, J. (2000). Trunk muscle activation in open stance and square stance tennis forehands. International journal of sports medicine, 21(5), 321-324. https://doi.org/10.1055/s-2000-3776

Kovacs, M. S. (2007). Tennis physiology: training the competitive athlete. Sports medicine, 37(3), 189-198. https://doi.org/10.1519/SSC.0b013e31818e5fbc

Kovacs, M. S.; Roeter, E. P., \& Ellenbecker, T. (2008). Efficient deceleration: The forgotten factor in tennis-specific training. Journal of strength \& Conditioning Research 30, 58-69.

https://doi.org/10.1519/SSC.0b013e31818e5fbc

Lees, A. (2003). Science and the major racket sports: a review. Journal of sports sciences, 21(9), 707-732. https://doi.org/10.1080/0264041031000140275

Lloyd, R. S.; Oliver, J. L.; Hughes, M. G., \& Williams, C. A. (2012). Age-related differences in the neural regulation of stretch-shortening cycle activities in male youths during maximal and sub-maximal hopping. Journal of Electromyography and Kinesiology, 22(1), 37-43.

https://doi.org/10.1016/j.jelekin.2011.09.008 
Pardos-Mainer, E.; Ustero-Pérez, O., y Gonzalo-Skok, O. (2017). Efectos de un entrenamiento pliométrico en extremidades superiores e inferiores en el rendimiento físico en jóvenes tenistas. RICYDE. Revista internacional de ciencias del deporte, 49(13), 225-243.

https://doi.org/10.5232/ricyde2017.04903

Markovic, G., \& Mikulic, P. (2010). Neuro-musculoskeletal and performance adaptations to lower-extremity plyometric training. Sports medicine, 40(10), 859-895. https://doi.org/10.2165/11318370-000000000-00000

Meylan, C. M.; Cronin, J. B.; Oliver, J. L.; Hopkins, W. G., \& Contreras, B. (2014). The effect of maturation on adaptations to strength training and detraining in 11-15-year-olds. Scandinavian journal of medicine \& science in sports, 24(3), e156-164. https://doi.org/10.1111/sms.12128

Murphy, A. P.; Duffield, R.; Kellett, A., \& Reid, M. (2015). The relationship of training load to physical-capacity changes during international tours in highperformance junior tennis players. International journal of sports physiology and performance, $10(2), 253-260$.

https://doi.org/10.1123/ijspp.2014-0038

Ozbar, N.; Ates, S., \& Agopyan, A. (2014). The effect of 8-week plyometric training on leg power, jump and sprint performance in female soccer players. Journal of strength and conditioning research / National Strength \& Conditioning Association, 28(10), 2888-2894.

https://doi.org/10.1519/JSC.0000000000000541

Ramirez-Campillo, R.; Alvarez, C.; Henriquez-Olguin, C.; Baez, E. B.; Martinez, C.; Andrade, D. C., y col. (2014). Effects of plyometric training on endurance and explosive strength performance in competitive middle- and long-distance runners. Journal of strength and conditioning research / National Strength \& Conditioning Association, 28(1), 97-104.

https://doi.org/10.1519/JSC.0b013e3182a1f44c

Ramirez-Campillo, R.; Andrade, D. C., \& Izquierdo, M. (2013). Effects of plyometric training volume and training surface on explosive strength. Journal of strength and conditioning research / National Strength \& Conditioning Association, $27(10), 2714-2722$.

https://doi.org/10.1519/JSC.0b013e318280c9e9

Ramirez-Campillo, R.; Gallardo, F.; Henriquez-Olguin, C.; Meylan, C. M.; Martinez, C.; Alvarez, C., y col. (2015). Effect of Vertical, Horizontal, and Combined Plyometric Training on Explosive, Balance, and Endurance Performance of Young Soccer Players. Journal of strength and conditioning research / National Strength \& Conditioning Association, 29(7), 1784-1795.

https://doi.org/10.1519/JSC.0000000000000827

Ramirez-Campillo, R.; Meylan, C.; Alvarez, C.; Henriquez-Olguin, C.; Martinez, C.; Canas-Jamett, R., y col. (2014). Effects of in-season low-volume high-intensity plyometric training on explosive actions and endurance of young soccer players. Journal of strength and conditioning research / National Strength \& Conditioning Association, 28(5), 1335-1342.

https://doi.org/10.1519/JSC.0000000000000284

Reid, M.; Crespo, M.; Santilli, L.; Miley, D., \& Dimmock, J. (2007). The importance of the International Tennis Federation's junior boys' circuit in the development of professional tennis players. Journal of sports sciences, 25(6), 667-672. https://doi.org/10.1080/02640410600811932

Reid, M.; Sibte, N.; Clarke, S., \& Whiteside, D. (2013). Protocols for the physiological assessment of tennis players. In Physiological tests for elite athletes (edited by R. Tanner \& C.J. Gore). Australia Human Kinetics. 
Pardos-Mainer, E.; Ustero-Pérez, O., y Gonzalo-Skok, O. (2017). Efectos de un entrenamiento pliométrico en extremidades superiores e inferiores en el rendimiento físico en jóvenes tenistas. RICYDE. Revista internacional de ciencias del deporte, 49(13), 225-243.

https://doi.org/10.5232/ricyde2017.04903

Rosch, D.; Hodgson, R.; Peterson, T. L.; Graf-Baumann, T.; Junge, A.; Chomiak, J., $y$ col. (2000). Assessment and evaluation of football performance. The American journal of sports medicine, 28(5 Suppl), S29-39. https://doi.org/10.1177/28.suppl_5.s-29

Rumpf, M. C.; Cronin, J. B.; Mohamad, I. N.; Mohamad, S.; Oliver, J. L., \& Hughes, M. G. (2015). The effect of resisted sprint training on maximum sprint kinetics and kinematics in youth. European Journal of Sport Science, 15(5), 374-381. https://doi.org/10.1080/17461391.2014.955125

Salonikidis, K., \& Zafeiridis, A. (2008). The effects of plyometric, tennis-drills, and combined training on reaction, lateral and linear speed, power, and strength in novice tennis players. Journal of strength and conditioning research / National Strength \& Conditioning Association, 22(1), 182-191. https://doi.org/10.1519/JSC.0b013e31815f57ad

Sannicandro, I.; Cofano, G.; Rosa, R. A., \& Piccinno, A. (2014). Balance training exercises decrease lower-limb strength asymmetry in young tennis players. Journal of sports science \& medicine, 13(2), 397-402.

Torres-Luque, G.; Sánchez-Pay, A.; Fernández-García, A., \& Palao, J. M. (2014). Características de la estructura temporal en tenis. Una revisión. (Characteristics of temporal structure in tennis. A review). Journal of Sport and Health Research, 6(2), 117-128.

Ulbricht, A.; Ferrauti, A., \& Fernández-Fernández, J. (2013). Conception for fitness testing and individualized training programs in the German Tennis Federation. Sports Orthopaedics and Traumatology, 29(3), 180-192. https://doi.org/10.1016/j.orthtr.2013.07.005 